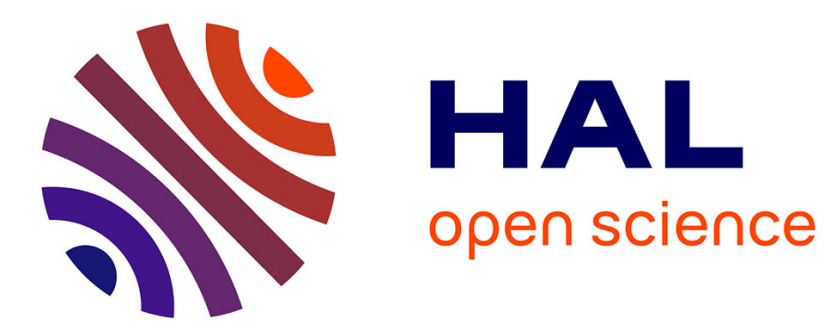

\title{
Structure of molecular packing probed by polarization-resolved nonlinear four-wave mixing and coherent anti-Stokes Raman-scattering microscopy
}

F.-Z. Bioud, P. Gasecka, Patrick Ferrand, H. Rigneault, J. Duboisset, S. Brasselet

\section{To cite this version:}

F.-Z. Bioud, P. Gasecka, Patrick Ferrand, H. Rigneault, J. Duboisset, et al.. Structure of molecular packing probed by polarization-resolved nonlinear four-wave mixing and coherent anti-Stokes Ramanscattering microscopy. Physical Review A : Atomic, molecular, and optical physics [1990-2015], 2014, 89 (1), pp.013836. 10.1103/PhysRevA.89.013836 . hal-00937566

\section{HAL Id: hal-00937566 https://hal.science/hal-00937566}

Submitted on 28 Jan 2014

HAL is a multi-disciplinary open access archive for the deposit and dissemination of scientific research documents, whether they are published or not. The documents may come from teaching and research institutions in France or abroad, or from public or private research centers.
L'archive ouverte pluridisciplinaire HAL, est destinée au dépôt et à la diffusion de documents scientifiques de niveau recherche, publiés ou non, émanant des établissements d'enseignement et de recherche français ou étrangers, des laboratoires publics ou privés. 


\title{
Structure of molecular packing probed by polarization-resolved nonlinear four-wave mixing and coherent anti-Stokes Raman-scattering microscopy
}

\author{
Fatma-Zohra Bioud, Paulina Gasecka, Patrick Ferrand, Hervé Rigneault, Julien Duboisset, ${ }^{*}$ and Sophie Brasselet ${ }^{\dagger}$ \\ Aix-Marseille Université, CNRS, Centrale Marseille, Institut Fresnel, UMR 7249, 13013 Marseille, France
}

(Received 9 May 2013; published 27 January 2014)

\begin{abstract}
We report a method that is able to provide refined structural information on molecular packing in biomolecular assemblies using polarization-resolved four-wave mixing and coherent anti-Stokes Raman-scattering microscopy. These third-order nonlinear processes allow quantifying high orders of symmetry which are exploited here to reveal a high level of detail in the angular disorder behavior at the molecular scale in lipid membranes.
\end{abstract}

DOI: 10.1103/PhysRevA.89.013836

PACS number(s): 42.65.Dr, 42.25.Ja, 42.65.An, 87.16.D-

\section{INTRODUCTION}

Structural organization of macromolecular assemblies (crystals, aggregates, membranes, and polymers) at the submicrometric scale is an important piece of information for material and life sciences. In particular, structural disorder in lipid membranes and lipid phases has been the focus of longterm interest in molecular physics and biology. This disorder, governed by local molecular interactions, conformational motions, and steric constraints, so far, has been approached by structural analysis tools, e.g., nuclear magnetic resonance [1], x-ray diffraction [2], and infrared spectroscopy [3]. These techniques, although able to provide precise quantitative information, however, still exhibit poor spatial resolution and are often not compatible with in vivo analysis in cells and tissues. Being able to report a high level of detail in the structural packing of molecules using optical microscopy would provide an interesting complementary alternative. This challenging issue can be addressed using polarization-resolved label-free nonlinear microscopy. Probing the orientationsensitive coupling between multiple optical excitation fields and transition dipole moments is, indeed, able to report molecular orientational behaviors in biological media at a submicrometric scale in a noninvasive way compatible with in vivo functional studies.

Whereas, polarization-sensitive second-harmonic generation microscopy is restricted to the analysis of noncentrosymmetric assemblies, such as collagen [4-7] and third-harmonic generation (THG) microscopy is specific to interfaces [8], polarization-resolved four-wave mixing (FWM) microscopy [9] and coherent anti-Stokes Raman-scattering (CARS) microscopy $[10,11]$ potentially are applicable to any kind of molecular assemblies' geometry. Another remarkable property of these contrasts is their high order of nonlinear interaction, making them sensitive to molecular angular distribution features up to the fourth order of symmetry [9,10,12]. This level of detail, which is a unique way to approach complex molecular organizations, so far, however, has not been used as functional information in bioimaging. One of the reasons for this is the difficulty to interpret data from polarization-resolved high-order nonlinear processes. Indeed, it requires the manipulation of a large number of nonlinear susceptibility tensor

\footnotetext{
*julien.duboisset@fresnel.fr

†'sophie.brasselet@fresnel.fr
}

components (both at the molecular and at the macroscopic scales) [13], which hampers a direct interpretation of the structural behavior. To quantify molecular order in lipid membranes, different strategies have been developed. Using the CARS contrast, the lipid $\mathrm{CH}_{2}$ symmetric stretching mode has been modeled using: (i) a single diagonal tensor term [14] or a $m m 2$ symmetry tensor $[15,16]$ without invoking orientational disorder, or (ii) a single diagonal tensor term undergoing a Gaussian orientational disorder [17]. In THG, a full calculation of the isolated lipid nonresonant tensor has been performed and has been distributed in a Gaussian disorder [8]. These models are far from being realistic due to the lack of knowledge on the lipids conformation, lipids composition, and macroscopic disorder present in complex samples, such as lipid multilayers in tissues. Clearly, there is a need to sort out the accessible parameters in these techniques and to be able to address structural order in samples where the molecular structure is not predetermined.

In this paper, we exploit the whole complexity of polarization-resolved FWM-CARS (denoted pFWM-pCARS) to measure model-independent information on the structural disorder present in lipid membranes. We reformulate the tensorial expression of the pFWM-pCARS macroscopic response by using a decomposition of the molecules into their individual bonds, acting as a collection of nonlinear radiation dipoles (nonlinear bond dipoles). This assembly of bond dipoles is, by construction, compatible with the symmetry properties of the microscopic hyperpolarizabilities involved in nonresonant (FWM) or vibration resonant (CARS) signals. We implement a pFWM-pCARS analysis to measure the macroscopic-scale angular distribution of these bond dipoles, denoted effective distribution. This distribution encompasses their molecularscale arrangement (possibly within different molecular conformations), the amplitudes of their nonlinear susceptibilities, and the macroscopic-scale disorder. We, furthermore, develop a symmetry decomposition of this distribution to interpret structural information in the sample in a rotational invariant approach independent of the distribution orientation $[6,18]$. Based on the measurements of the second and fourth orders of symmetries of the distribution, which are accessible by pFWM-pCARS, we illustrate how the determination of the high order of symmetry permits revealing details on orientational disorder in multilamellar vesicles (MLVs) and myelin in tissues without having to infer any a priori knowledge of the precise molecular composition, conformation, and statistical disorder present in the sample. 


\section{SYMMETRY ORDERS ACCESSIBLE BY POLARIZATION-RESOLVED FWM-CARS}

\section{A. Model}

The calculation of the polarized nonlinear responses from molecular assemblies generally relies on the expression of the nonlinear hyperpolarizability tensor of single molecules, averaged macroscopically over the orientational disorder within the focal volume of excitation, assuming negligible interaction between the molecules $[12,13]$.

The electronic third-order hyperpolarizability of lipid molecules is at the origin of FWM responses in lipid membranes. At the single lipid scale, the hyperpolarizability tensor $\chi_{i j k l}^{(3)}$ [defined in the molecular local frame $(x, y, z)$, Fig. 1(a)] has been obtained using a bond additive model, which supposes that all nonlinear radiation sources of individual molecular bonds add up coherently [8]. Similarly, for resonant CARS-polarized responses, hyperpolarizabilities associated with the resonant vibrational bonds can be deduced from the symmetry of their corresponding vibrational modes $[13,19,20]$. Whereas, a complete model can address the case where both resonant and nonresonant contributions to the CARS signal are present $[10,13]$, here we address cases where the resonance is dominant (and, therefore, the nonresonant contribution of the CARS tensor is negligible). This is, in particular, the case for the $\mathrm{CH}_{2}$ stretching mode in lipids [21].

As mentioned above, the macroscopic nonlinear tensor $\chi_{I J K L}^{(3)}$ of the lipid assembly [defined in the macroscopic frame $(X, Y, Z)$ where $Z$ is the propagation direction and $(X, Y)$ is the sample plane, Fig. 1(b)] is obtained by a statistical orientational averaging over a given normalized distribution function

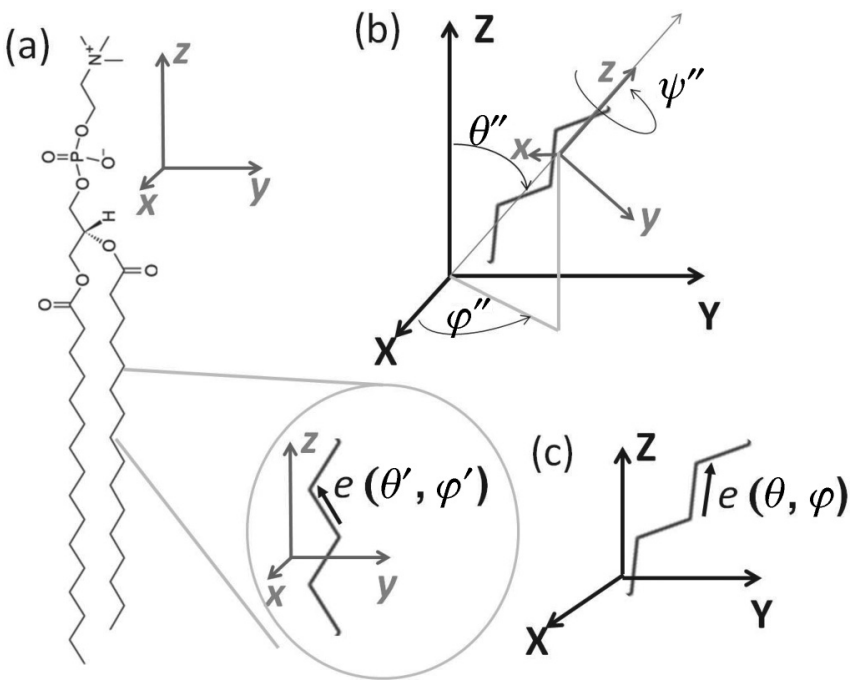

FIG. 1. (a) Schematic of a lipid alkyl chain in the lipid molecular frame $(x, y, z)$ and one nonlinear dipole e involved in the lipid nonlinear response. (b) Schematic of a lipid alkyl chain molecule in the macroscopic frame $(X, Y, Z)$, indicating the $\Omega^{\prime \prime}$ set of angles. $(X, Y)$ is the sample plane which contains the incident field polarizations propagating in the $Z$ direction. (c) Schematic of the $\Omega^{\prime}$ and $\Omega$ sets of angles defining the orientation of the nonlinear dipoles e, respectively, in the molecular and macroscopic frames.
$h\left(\Omega^{\prime \prime}\right)$,

$$
\chi_{I J K L}^{(3)}=N \int \sum_{i j k l}(\mathbf{I} \cdot \mathbf{i})(\mathbf{J} \cdot \mathbf{j})(\mathbf{K} \cdot \mathbf{k})(\mathbf{L} \cdot \mathbf{l}) \chi_{i j k l}^{(3)} h\left(\Omega^{\prime \prime}\right) d \Omega^{\prime \prime},
$$

with $N$ as the molecular density and $(I, J, K, L) \in(X, Y, Z)$. $\Omega^{\prime \prime}=\left(\theta^{\prime \prime}, \phi^{\prime \prime}, \psi^{\prime \prime}\right)$ is the Euler set of the angle defining the lipid molecule orientation in the macroscopic frame [Fig. 1(b)], and $d \Omega^{\prime \prime}=\sin \theta^{\prime \prime} d \theta^{\prime \prime} d \varphi^{\prime \prime} d \psi^{\prime \prime}$. The distribution function generally is chosen as a Gaussian function of width $\sigma$ lying in the $(X, Y)$ sample plane: $h\left(\Omega^{\prime \prime}\right) \propto \exp \left[-\left(\theta^{\prime \prime}-\right.\right.$ $\left.\left.\left\langle\theta^{\prime \prime}\right\rangle\right)^{2} / 2 \sigma^{2}\right]$ with $X$ as the symmetry axis of this distribution $[8,17]$.

If applied to the structural investigation of lipid membranes, this model, however, presents some limitations: (i) It requires the knowledge of the hyperpolarizability tensorial components $\chi_{i j k l}^{(3)}$, which is, in practice, delicate to obtain; (ii) it supposes that the lipids all have the same conformation, which is unlikely to occur in artificial and cell membranes; (iii) it applies to membranes composed of a single type of lipid, which is not the case in cells and tissues; and (iv) it supposes that the lipid orientational distribution follows a known shape, which requires an a priori assumption on the molecular interactions taking place in the medium.

In the present approach, we propose decomposing the nonlinear polarized response into its individual nonlinearinduced polarizations, originating from nonlinear sources at the single bond level. The single bond response is described based on its tensorial symmetry properties for which principal vectors can be defined. The directions of these vectors, denoted e [Fig. 1(b)], follow the axes of the bond invariant transformations, and their amplitudes are characteristic of the considered bond, deduced from quantum mechanics or nonlinear bond energy expansion [8]. These vectors, called bond dipoles in what follows, therefore, are compatible with the symmetry of the molecular structures involved in the nonlinear process.

The molecular lipid tensor can be built up based on the sum of the individual bond dipoles' nonlinear contributions. In the molecular frame $(x, y, z)$, the vectors $\mathbf{e}$ are distributed along a normalized distribution function $g\left(\Omega^{\prime}\right)$, encompassing their orientation $\Omega^{\prime}=\left(\theta^{\prime}, \varphi^{\prime}\right)$ [Fig. 1(a)] and their hyperpolarizability relative amplitudes such that $g\left(\Omega^{\prime}\right)$ is normalized

$$
\chi_{i j k l}^{(3)}=\gamma \int_{\Omega^{\prime}}(\mathbf{i} \cdot \mathbf{e})(\mathbf{j} \cdot \mathbf{e})(\mathbf{k} \cdot \mathbf{e})(\mathbf{I} \cdot \mathbf{e}) g\left(\Omega^{\prime}\right) d \Omega^{\prime},
$$

with $\gamma$ as the norm of the molecule hyperpolarizability. Note that, in the case of a crystalline molecule, the distribution $g\left(\Omega^{\prime}\right)$ would be made of Dirac functions, but also can include orientational averaging at this stage. Note that this expression leads, in principle, to the same multipolar $\chi_{i j k l}^{(3)}$ tensor structure obtained from an additive bond model since the symmetry properties of the bond dipoles' assembly is contained in the properties of the $g\left(\Omega^{\prime}\right)$ function. Following Eq. (2), the macroscopic tensor then 
becomes

$$
\begin{aligned}
\chi_{I J K L}^{(3)}= & N \gamma \int_{\Omega^{\prime \prime}} \int_{\Omega^{\prime}} \sum_{i j k l}(\mathbf{I} \cdot \mathbf{i})(\mathbf{i} \cdot \mathbf{e})(\mathbf{J} \cdot \mathbf{j})(\mathbf{j} \cdot \mathbf{e}) \\
& \times(\mathbf{K} \cdot \mathbf{k})(\mathbf{k} \cdot \mathbf{e})(\mathbf{L} \cdot \mathbf{I})(\mathbf{I} \cdot \mathbf{e}) g\left(\Omega^{\prime}\right) h\left(\Omega^{\prime \prime}\right) d \Omega^{\prime} d \Omega^{\prime \prime} .
\end{aligned}
$$

A more explicit expression of the projection factors in Eq. (3) can help simplify this expression. Since the products, such as $(\mathbf{I} \cdot \mathbf{i})(\mathbf{i} \cdot \mathbf{e})$ refer to the orientation of the $\mathbf{e}$ vector in the macroscopic $(X, Y, Z)$ frame, they can be reduced as $(\mathbf{I} \cdot \mathbf{e})$, which is a function of $\Omega=(\theta, \varphi)$, defining the orientation of $\mathbf{e}$ in this macroscopic frame [Fig. 1(c)]. Since $\Omega$ depends implicitly on $\left(\Omega^{\prime}, \Omega^{\prime \prime}\right)$, rewriting Eq. (3) as a dependence of $\Omega$ and $\Omega^{\prime \prime}$ leads to

$$
\chi_{I J K L}^{(3)}=N \gamma \int_{\Omega}(\mathbf{I} \cdot \mathbf{e})(\mathbf{J} \cdot \mathbf{e})(\mathbf{K} \cdot \mathbf{e})(\mathbf{L} \cdot \mathbf{e}) f(\Omega) d \Omega,
$$

with

$$
f(\Omega)=\int_{\Omega^{\prime \prime}} g\left(\Omega, \Omega^{\prime \prime}\right) h\left(\Omega^{\prime \prime}\right) d \Omega^{\prime \prime} .
$$

$f(\Omega)$ can be interpreted as the distribution function of orientations $\Omega$ of the e vectors in the macroscopic frame. It can easily be visualized in a two-dimensional (2D) model where bond dipoles and molecules would be defined on a single plane $(X, Y)$ : Indeed, in this case, the $(\mathbf{I} \cdot \mathbf{e})$ products are functions of $\left(\varphi^{\prime}+\varphi^{\prime \prime}\right)$, and therefore, using the change in variable $\varphi=\varphi^{\prime}+\varphi^{\prime \prime}$ leads to

$$
\chi_{I J K L}^{(3), 2 D}=N \gamma \int_{\varphi}(\mathbf{I} \cdot \mathbf{e})(\mathbf{J} \cdot \mathbf{e})(\mathbf{K} \cdot \mathbf{e})(\mathbf{L} \cdot \mathbf{e}) f^{2 D}(\varphi) d \varphi,
$$

where the functions now are written in a $2 \mathrm{D}$ frame using a $2 D$ upper index notation. In this context,

$$
f^{2 D}(\varphi)=\int_{\varphi^{\prime \prime}} g^{2 D}\left(\varphi-\varphi^{\prime \prime}\right) h^{2 D}\left(\varphi^{\prime \prime}\right) d \varphi^{\prime \prime}=g * h .
$$

From Eq. (7), it appears, indeed, intuitive that the global distribution of lipids of multipolar nature is the convolution of the lipid axis distribution by the bond dipoles' distribution constituting these molecules.

Finally, note that this approach supposes that all index permutations are allowed within the microscopic tensor components, which is valid for nonresonant FWM, but only under specific conditions for CARS (for instance, in lipids, for the symmetric stretching $\mathrm{CH}_{2}-\mathrm{CD}_{2}$ mode, which belongs to the $\mathrm{mm} 2$ symmetry group, with no electronic resonance involved) [13].

\section{B. Effective distribution function}

In what follows, we, furthermore, demonstrate how, when transverse incoming polarizations are used, such a model can lead to a reduced expression where only one angular variable $\varphi$ is needed. We consider the case where the incident polarizations $\mathbf{E}$, involved in the nonlinear coupling, lie in the transverse $(X, Y)$ sample plane, therefore, $E_{Z}=0$, and there are no $Z$ components involved in the tensorial coupling with the fields. In practice, strong focusing conditions involve an $E_{Z}$ component which can raise up to $40 \%$ in amplitude compared to in-plane components [22]. This contribution will be neglected here since, in the case of the type of distributions studied here, only a strong out-of-plane tilt of the sample can lead to a noticeable bias in the nonlinear polarized measurements $[23,24]$. Each remaining in-plane tensorial component of the previous expression can then be expressed more specifically, for instance,

$$
\chi_{X X X X}^{(3)}=N \gamma \int_{\theta} \int_{\varphi} \sin ^{5} \theta \cos ^{4} \varphi f(\theta, \varphi) d \varphi d \theta,
$$

and can be expressed similarly for the other components. A $\theta$ integration is, therefore, possible, which introduces a further simplification using

$$
p(\varphi)=\int_{\theta} \sin ^{5} \theta f(\theta, \varphi) d \theta .
$$

The macroscopic tensor components become, therefore,

$$
\begin{aligned}
& \chi_{X X X X}=N \gamma \int_{0}^{2 \pi} \cos ^{4} \varphi p(\varphi) d \varphi, \\
& \chi_{X X X Y}=N \gamma \int_{0}^{2 \pi} \cos ^{3} \varphi \sin \varphi p(\varphi) d \varphi, \\
& \chi_{X Y X Y}=N \gamma \int_{0}^{2 \pi} \cos ^{2} \varphi \sin ^{2} \varphi p(\varphi) d \varphi, \\
& \chi_{X Y Y Y}=N \gamma \int_{0}^{2 \pi} \sin ^{3} \varphi \cos \varphi p(\varphi) d \varphi, \\
& \chi_{Y Y Y Y}=N \gamma \int_{0}^{2 \pi} \sin ^{4} \varphi p(\varphi) d \varphi .
\end{aligned}
$$

The nonlinear response finally depends on the function $p(\varphi)$ characterizing the orientation of the bond dipoles. This function is not a $2 \mathrm{D}$ section nor a geometric $2 \mathrm{D}$ projection of their initial three-dimensional (3D) distribution $f(\Omega)$, but rather the result of an out-of-plane integration of the 3D nonlinear coupling involving the bond dipoles' population. $p(\varphi)$ is, therefore, an effective distribution function characterizing the orientational behavior of these bond dipoles. Note that this approach finally requires that the initial distribution function $h\left(\Omega^{\prime \prime}\right)$ lies as much as possible on the sample plane, which is generally the case in the frame of polarization-resolved studies.

\section{Symmetry decomposition}

In order to exhibit the symmetry properties of the tensor defined in Eq. (10), a spherical expansion of the 3D distribution function $f(\Omega)$ is introduced

$$
f(\Omega)=\sum_{m, l} f_{m, l} Y_{m}^{l}(\Omega),
$$

where the $f_{m, l}$ coefficients are the components of $f(\Omega)$ on the real spherical basis functions $Y_{m}^{l}(\Omega)$ of symmetry order $l$ (with $-l \leqslant m \leqslant l)$,

$$
\begin{aligned}
Y_{m}^{l}(\theta, \varphi) & =N_{(m, l)} P_{m}^{l}(\cos \theta) \cos (m \varphi) \quad \text { for } m>0 \\
& =N_{(|m|, l)} P_{|m|}^{l}(\cos \theta) \sin (|m| \varphi) \text { for } m<0 \\
& =Y_{0}^{l}(\theta, \varphi) \quad \text { for } m=0,
\end{aligned}
$$

with $P_{m}^{l}(\cos \theta)$ as the associated Legendre polynomials and $N_{(m, l)}=\sqrt{\frac{(2 l+1)(l-m) !}{\pi(l+m) !}}$. 
Applying Eq. (11) to Eq. (9) leads to a symmetry-order decomposition of $p(\varphi)$,

$$
\begin{aligned}
p(\varphi)= & \sum_{m, l} \int_{\theta} \sin ^{5} \theta f_{m, l} Y_{m}^{l}(\theta, \varphi) d \theta=\left[\sum_{l} \int_{\theta} \sin ^{5} \theta f_{0, l} Y_{0}^{l}(\theta) d \theta\right]+\sum_{m>0} \cos (m \varphi)\left[\sum_{l} \int_{\theta} \sin ^{5} \theta f_{m, l} N_{(m, l)} P_{m}^{l}(\cos \theta) d \theta\right] \\
& +\sum_{m<0} \sin (|m| \varphi)\left[\sum_{l} \int_{\theta} \sin ^{5} \theta f_{|m|, l} N_{(|m|, l)} P_{|m|}^{l}(\cos \theta) d \theta\right]=p_{0}+\sum_{n} p_{n} \cos (n \varphi)+q_{n} \sin (n \varphi),
\end{aligned}
$$

with $n$ as a positive integer.

The resulting order parameters $\left(p_{n}, q_{n}\right)$ can be interpreted in a similar way as the order parameters $\left\langle P_{n}[\cos (\theta)]\right\rangle$ derived from Legendre polynomials, used in structural analyses $[1,3]$. Note, however, that the more general approach introduced here allows us to reveal possible distributions which do not exhibit a cylindrical symmetry, which is not the case using pure Legendre polynomial decompositions.

The retrieved order parameters finally encompass both the multipolar nature of the individual lipid molecules and their 3D angular distributions. Although these two properties cannot be extracted independently, rich structural information can be obtained from them without the need of a priori models on the molecular structure nor on their macroscopic disorder.

Finally, in Eq. (10), one can notice that the macroscopic tensors involved in FWM-CARS processes only involve $\varphi$ functions of even orders up to the fourth order, therefore, only even orders $n=0,2,4$ of $p(\varphi)$ are involved, and the retrieved distribution will be reduced to

$$
\begin{aligned}
\tilde{p}(\varphi)= & p_{0}+p_{2} \cos (2 \varphi)+q_{2} \sin (2 \varphi)+p_{4} \cos (4 \varphi) \\
& +q_{4} \sin (4 \varphi) .
\end{aligned}
$$

By using $\tilde{p}(\varphi)$, we finally get the following relations between the components of the macroscopic nonlinear susceptibility and the truncated angular distribution function parameters:

$$
\begin{aligned}
& \chi_{X X X X}=N \gamma \frac{\pi\left(6 p_{0}+4 p_{2}+p_{4}\right)}{8}, \\
& \chi_{X X X Y}=N \gamma \frac{\pi\left(2 q_{2}+q_{4}\right)}{8}, \\
& \chi_{X Y X Y}=N \gamma \frac{\pi\left(2 p_{0}-p_{4}\right)}{8}, \\
& \chi_{X Y Y Y}=N \gamma \frac{\pi\left(2 q_{2}-q_{4}\right)}{8}, \\
& \chi_{Y Y Y Y}=N \gamma \frac{\pi\left(6 p_{0}-4 p_{2}+p_{4}\right)}{8} .
\end{aligned}
$$

Polarization-resolved nonlinear FWM and CARS signals, which depend on these coefficients, should, therefore, allow access to the order parameters $\left(p_{n}, q_{n}\right)$ of second and fourth symmetries and, therefore, to structural information in the investigated sample.

\section{Deduction of order parameters $\left(p_{n}, q_{n}\right)$ from FWM-CARS-polarized responses}

The nonlinear-induced polarization originates from the coupling between the macroscopic nonlinear susceptibility tensor and the incoming fundamental fields,

$$
\mathbf{P}^{(3)}\left(\omega_{a s}\right)=\sum_{I J K L} \chi_{I J K L}^{(3)} \mathbf{E}\left(\omega_{p}\right)_{J} \mathbf{E}\left(\omega_{p}\right)_{K} \mathbf{E}^{*}\left(\omega_{s}\right)_{L},
$$

where $(I, J, K, L) \in(X, Y, Z)$. The vectors $\mathbf{E}_{p, s}$ are the incident electrical fields for the pump and Stokes frequencies $\omega_{p}$ and $\omega_{s}$, and $\omega_{a s}=2 \omega_{p}-\omega_{s}$. Using electric fields polarized in the $(X, Y)$ plane, Eq. (16) can be developed into

$$
\begin{aligned}
P_{X}= & \chi_{X X X X} E_{X} E_{X} E_{X}+3 \chi_{X X X Y} E_{X} E_{X} E_{Y} \\
& +3 \chi_{X Y X Y} E_{Y} E_{X} E_{Y}+\chi_{X Y Y Y} E_{Y} E_{Y} E_{Y}, \\
P_{Y}= & \chi_{Y X X X} E_{X} E_{X} E_{X}+3 \chi_{Y X X Y} E_{X} E_{X} E_{Y} \\
& +3 \chi_{Y Y X Y} E_{Y} E_{X} E_{Y}+\chi_{Y Y Y Y} E_{Y} E_{Y} E_{Y} .
\end{aligned}
$$

The total intensity is, thus,

$$
I \propto\left|P_{X}\right|^{2}+\left|P_{Y}\right|^{2}
$$

Note that possible polarization scrambling due to the high aperture collection is not perceptible here since the detection is unpolarized. This allows the use of high numerical aperture objectives for the signal collection without a necessary correction factor.

In polarized FWM-CARS, the pump and Stokes electric fields all are polarized linearly along the same direction, defined by angle $\alpha$ relative to the $X$ axis: $\mathbf{E}_{s, p}=\left(E_{X}=\right.$ $\left.E_{s, p} \cos \alpha, E_{Y}=E_{s, p} \sin \alpha, E_{Z}=0\right)$ with $E_{s, p}$ as the field amplitude of the Stokes-pump beam. The intensity then is expressed as a function of $\alpha$ and can be rearranged to exhibit Fourier coefficients $a_{n}$ and $b_{n}$ for $n=0,2,4,6$,

$$
\begin{aligned}
I= & a_{0}+a_{2} \cos (2 \alpha)+a_{4} \cos (4 \alpha)+a_{6} \cos (6 \alpha) \\
& +b_{2} \sin (2 \alpha)+b_{4} \sin (4 \alpha)+b_{6} \sin (6 \alpha),
\end{aligned}
$$

with

$$
\begin{aligned}
a_{0}= & \frac{E}{16}\left(5 \chi_{1}^{2}+14 \chi_{2}^{2}+18 \chi_{4}^{2}+14 \chi_{8}^{2}+5 \chi_{9}^{2}\right. \\
& \left.+12 \chi_{2} \chi_{8}+6 \chi_{1} \chi_{4}+6 \chi_{4} \chi_{9}\right), \\
a_{2}= & \frac{E}{32}\left(15 \chi_{1}^{2}+6 \chi_{2}^{2}-15 \chi_{9}^{2}-12 \chi_{8}^{2}+6 \chi_{4} \chi_{1}-6 \chi_{4} \chi_{9}\right), \\
a_{4}= & \frac{E}{16}\left(3 \chi_{1}^{2}-6 \chi_{2}^{2}-6 \chi_{8}^{2}-18 \chi_{4}^{2}+3 \chi_{9}^{2}\right. \\
& \left.-6 \chi_{1} \chi_{4}-12 \chi_{2} \chi_{8}-6 \chi_{4} \chi_{9}\right), \\
a_{6}= & \frac{E}{32}\left(\chi_{1}^{2}-2 \chi_{2}^{2}+8 \chi_{8}^{2}-\chi_{9}^{2} / 16-6 \chi_{4} \chi_{1}+6 \chi_{4} \chi_{9}\right), \\
b_{2}= & \frac{E}{16}\left(15 \chi_{1} \chi_{2}+42 \chi_{2} \chi_{4}+3 \chi_{1} \chi_{8}+3 \chi_{2} \chi_{9}\right. \\
& \left.+42 \chi_{4} \chi_{8}+15 \chi_{8} \chi_{9}\right),
\end{aligned}
$$




$$
\begin{aligned}
& b_{4}=\frac{E}{4}\left(3 \chi_{1} \chi_{2}+3 \chi_{2} \chi_{4}-3 \chi_{4} \chi_{8}-3 \chi_{8} \chi_{9}\right), \\
& b_{6}=\frac{E}{16}\left(3 \chi_{1} \chi_{2}-6 \chi_{2} \chi_{4}-\chi_{1} \chi_{8}-\chi_{2} \chi_{9}-6 \chi_{4} \chi_{8}+3 \chi_{8} \chi_{9}\right),
\end{aligned}
$$

with $E=E_{p}^{2} E_{s}$ and

$$
\begin{aligned}
& \chi_{1}=\chi_{X X X X}, \quad \chi_{2}=\chi_{X X X Y}, \quad \chi_{4}=\chi_{X Y X Y}, \\
& \chi_{8}=\chi_{X Y Y Y}, \quad \chi_{9}=\chi_{Y Y Y Y} .
\end{aligned}
$$

Combining Eqs. (15), (20), and (21), one can rewrite the $\left(a_{n}, b_{n}\right)$ Fourier coefficients as functions of the $\left(p_{n}, q_{n}\right)$ order parameters,

$$
\begin{aligned}
& a_{0}=\frac{A}{64}\left(36+10 p_{2}^{2}+10 q_{2}^{2}+p_{4}^{2}+q_{4}^{2}\right), \\
& a_{2}=\frac{A}{32}\left(24 p_{2}+3 p_{2} p_{4}+3 q_{2} q_{4}\right), \\
& a_{4}=\frac{3 A}{32}\left(p_{2}^{2}-q_{2}^{2}+2 p_{4}\right), \\
& a_{6}=\frac{A}{32}\left(p_{2} p_{4}-q_{2} q_{4}\right), \\
& b_{2}=\frac{A}{32}\left(24 q_{2}+3 p_{2} q_{4}-32 p_{4} q_{2}\right), \\
& b_{4}=\frac{3 A}{16}\left(2 q_{4}+p_{2} q_{2}\right), \\
& b_{6}=\frac{A}{32}\left(p_{2} q_{4}+p_{4} q_{2}\right) .
\end{aligned}
$$

with $A=N^{2} \gamma^{2} E$.

\section{EFFECTIVE DISTRIBUTION MODEL}

\section{A. Comparison with a molecular distribution approach}

Even though they measure the same polarized signals, the approaches given in Eqs. (1) [searching for a molecular distribution function $h(\Omega)$ ] and (4) [searching for an effective nonlinear dipoles function $p(\varphi)$ ] differ in interpretation. To compare these two approaches, we study the case of the $\mathrm{pFWM}$ response from identical lipids oriented within a known distribution function. The lipid molecule modeled for this theoretical study is 1,2-dimyristoyl-sn-glycero-3phosphorylcholine, which has recently been documented in THG [8] and structurally is similar to the lipids used in this paper.

In the case of nonresonant FWM, the molecular lipid tensor $\chi_{i j k l}^{(3)}$ is expected to have a similar structure as THG with dominant diagonal terms $\chi_{i i i i}^{(3)}$ which scale at about three times the off-diagonal terms $\chi_{i i j j}^{(3)}$ and negligible $\chi_{i i i j}^{(3)}$ values with $(i, j) \in(x, y, z)$ [8]. The lipid molecule distribution $h(\Omega)$ generally is assumed to follow a Gaussian function of width $\sigma$ lying along the $X$ macroscopic axis, the molecules first being averaged over their main-axis rotation angle $\psi$. Using Eqs. (1) and (16)-(18), the simulated FWM polarization response $I(\alpha)$ is calculated and is plotted in Fig. 2 in a polar plot representation for three different cases: a single molecule lying along $X$ [Fig. 2(a)], a narrow Gaussian function with (a)
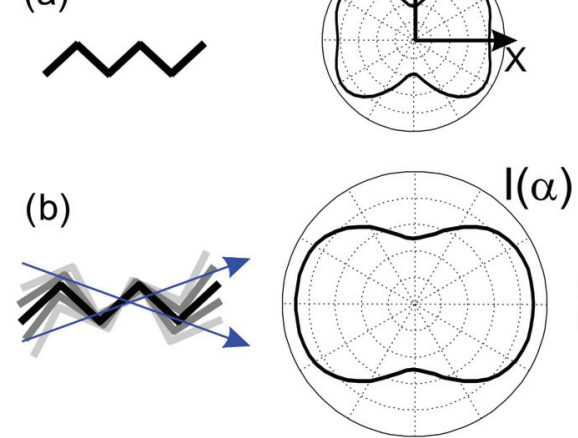

(c)
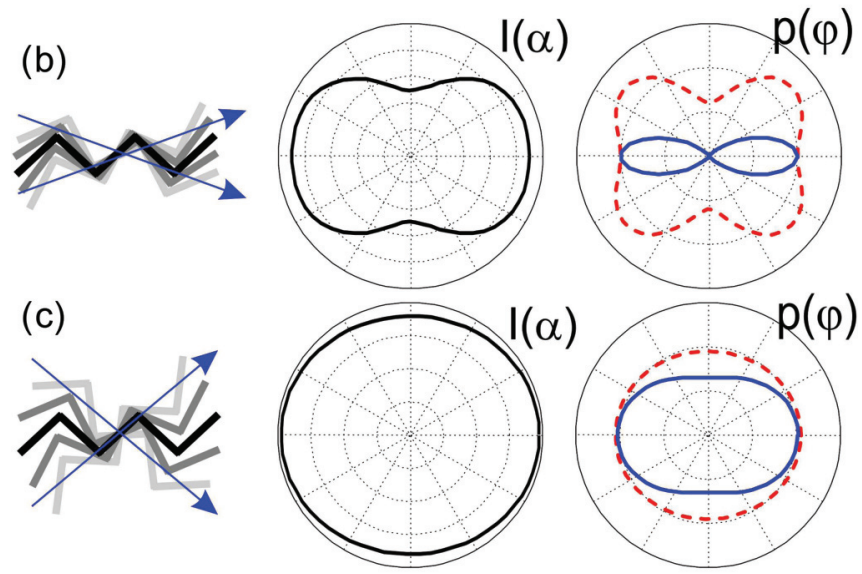

Lipids distribution

Bond dipoles' distribution

FIG. 2. (Color online) (a) Polarization-resolved FWM intensity $[I(\alpha)$ in a polar representation] of a single lipid molecule lying along $X$. The $\chi_{i j k l}^{(3)}$ tensor components are modeled as described in the text following Eq. (2) and Ref. [8]). (b) Left: Gaussian distribution of lipid molecules with a width of $\sigma=20^{\circ}$. Middle: FWM intensity deduced from this model. Right: polar representation of the distribution, dashed line: $\tilde{p}(\varphi)$ used for bond dipoles, continuous line: 2D section $h(\varphi)=\exp \left(-\varphi^{2} / 2 \sigma^{2}\right)$ used for lipid molecules. (c) The same analysis for a large Gaussian distribution of molecules with $\sigma=60^{\circ}$.

$\sigma=20^{\circ}$ [Fig. 2(b)], and a large distribution with $\sigma=60^{\circ}$ [Fig. 2(c)]. One can see from the FWM-polarization responses that the more disorder is introduced, the less contrasted this polarization response is, as expected.

The modeled polarization responses then are used for a retrieval of the bond dipoles' effective distribution function $\tilde{p}(\varphi)$ using Eq. (22) (see Sec. IV B for the details on the reconstruction method). The retrieved $\tilde{p}(\varphi)$ is represented in a polar plot representation in Figs. 2(b) and 2(c). For a highly ordered sample, the multipolar structure of the molecule itself is visible in the reconstructed function $\tilde{p}(\varphi)$, which is expected since the symmetry of this structure is included in the deduced order parameters. We recognize, in particular, in Figs. 2(b) and 2(c), the main directions of the CC bonds in the macroscopic response since these bonds do not lose their orientational order in these cases. When a higher disorder is applied [Fig. 2(c)], this molecular multipolar structure is smoothed by the orientational averaging and is less visible.

Finally, in the first approach, the interpretation of $I(\alpha)$ would require knowing the exact molecular tensorial structure $\left(\chi_{i j k l}^{(3)}\right.$ coefficients) as well as the macroscopic angular distribution of the molecules $[h(\Omega)$ model], leading to a measurement of the disorder parameter $\sigma$, which is model dependent $[8,17]$. In the second approach addressed in this paper, no a priori knowledge is introduced on the molecular scale nor on the macroscopic distribution: The measured order parameters $\left(p_{n}, q_{n}\right)$ allow quantifying structural information in 
a general way based on the bond dipoles' disorder present in the focal volume assembly.

In the case of resonant CARS, a different molecular hyperpolarizability tensor is involved. For a dominant vibrational $\mathrm{CH}_{2}$ resonance (symmetric $m m 2$ symmetry), this microscopic tensor contains the nonvanishing components $\chi_{i i i i}^{(3)}$ and $\chi_{i i j j}^{(3)}$ $\left[(i, j) \in(x, y, z)\right.$ being the local frame of the $\mathrm{CH}_{2}$ bond lying in the $(x, y)$ plane] with all permutations allowed [13]. Interpretation of polarized CARS responses have essentially been based on a simplification of the $\mathrm{CH}_{2}$ resonance tensor using $\chi_{x x x x}^{(3)}$ as a major contribution along the bond symmetry axis and a Gaussian distribution of the lipid molecules [17]. The resulting polarization responses would have, in this case, similar interpretations since this corresponds to unique sets of dipoles arranged along the $\mathrm{CH}_{2}$ main axis of symmetry: Since this axis is perpendicular to the lipid bond, the effective distribution $\tilde{p}(\varphi)$ would resemble a Gaussian reconstruction pointing at $90^{\circ}$ relative to the lipid membrane contour.

\section{B. Interpretation of order parameters in lipids' assemblies}

The $\tilde{p}(\varphi)$ function contains rich information on the symmetry decomposition of the complete effective distribution $p(\varphi)$. Since the second and fourth orders of symmetry give only a truncated view of this function, $p(\varphi)$ cannot be determined unambiguously, however, the access to these two orders already is sufficient to unravel information that would not be accessible with lower orders of symmetry. To illustrate this gain in information, simple distribution functions $p(\varphi)$ are considered in this part and are decomposed in symmetry orders. Following Eq. (9), these symmetry orders can be deduced from

$$
\begin{aligned}
& p_{0}=\frac{1}{2 \pi} \int p(\varphi) d \varphi, \\
& p_{n}=\frac{1}{\pi} \int p(\varphi) \cos (n \varphi) d \varphi, \\
& q_{n}=\frac{1}{\pi} \int p(\varphi) \sin (n \varphi) d \varphi .
\end{aligned}
$$

In what follows, the $\left(p_{n}, q_{n}\right)$ coefficients are normalized to $p_{0}$, which we set equal to $p_{0}=1$. These coefficients can be redistributed to define the magnitude of this $n$ th-order component $\left(S_{n}\right)$ and its orientation $\left(\varphi_{n}\right)$,

$$
\begin{aligned}
S_{n} & =\sqrt{\left(p_{n}^{2}+q_{n}^{2}\right)}, \\
\varphi_{n} & = \begin{cases}\frac{1}{n} \arctan \left(q_{n} / p_{n}\right), & \text { if } p_{n}>0, \\
\frac{1}{n} \arctan \left(q_{n} / p_{n}\right)+\frac{\pi}{n}, & \text { else. }\end{cases}
\end{aligned}
$$

These terms allow discriminating the invariant symmetry information $S_{n}$ from the more experiment-specific orientation information $\varphi_{n}$. Every distribution function read out by a pFWM-pCARS process will, thus, be represented by its symmetry spectrum $\left(S_{2}, S_{4}\right)$.

The $p(\varphi)$ functions studied in this part are inspired from simplified lipid organizations, such as schematically represented in Fig. 2.

As mentioned above, the FWM signal originating from this assembly would originate from a third-order hyperpolarizability tensor dominated by a trans alkyl chain symmetry (a)

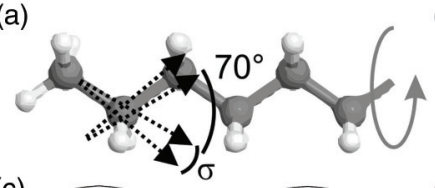

(b)

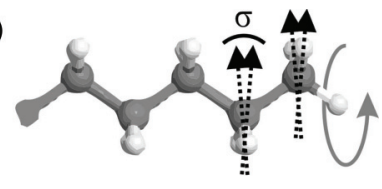

(c)

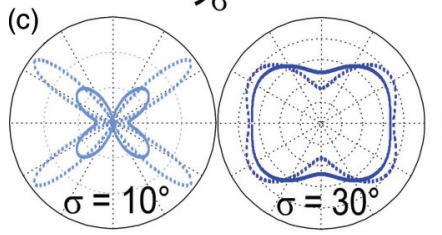

(e)
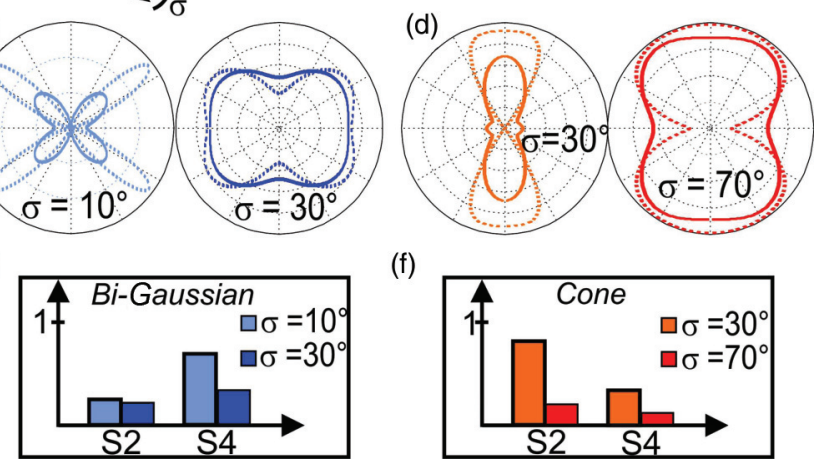

(f)

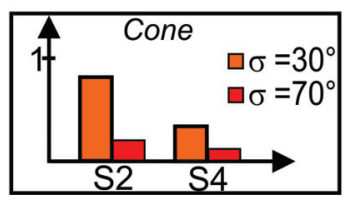

FIG. 3. (Color online) Schematic of a lipid chain, pointing out the dominant nonlinear bond dipoles' directions (arrows) for (a) nonresonant FWM (CC bonds) and (b) resonant CARS ( $\mathrm{CH}$ bonds). (c) Polar representation of two bi-Gaussian functions $p(\varphi)$ of different widths (dashed lines) with their $\left(S_{2}, S_{4}\right)$ circular reconstruction $\tilde{p}(\varphi)$ (continuous lines). (d) Similar representations for a cone $(a=6)$. (e) and (f) Symmetry spectra of the functions represented in (c) and (d), respectively.

[8], pictured by two main directions of the CC bonds with an interangle of about $70^{\circ}$ [Fig. 3(a)]. Assuming that the angular disorder experienced by the lipids is modeled by a Gaussian function $[8,17]$ centered on $\varphi_{0}$ and of width $\sigma$, this disorder will cause the associated nonlinear bond dipoles to lie within a bi-Gaussian function,

$$
p(\varphi) \propto e^{-\left(\varphi-\varphi_{0}-\varepsilon / 2\right)^{2} / 2 \sigma^{2}}+e^{-\left(\varphi-\varphi_{0}+\varepsilon / 2\right)^{2} / 2 \sigma^{2}}
$$

made of two Gaussian functions distant by about $\varepsilon \sim 70^{\circ}$ [Fig. 3(c)].

In contrast, the CARS susceptibility of the lipids at their symmetric $\mathrm{CH}_{2}$-stretching resonance is governed by the perpendicular orientation of the $\mathrm{CH}_{2}$ vibrational bonds to the alkyl chain [Fig. 3(b)], leading to an effective distribution $p(\varphi)$ of the bond dipoles which could simply resemble a Gaussian function. In order to provide a larger symmetry spectrum for this distribution, we introduce a super-Gaussian function centered on $\varphi_{0}$ and of width $\sigma$ [Fig. 3(d)],

$$
p(\varphi) \propto e^{-\left(\left|\varphi-\varphi_{0}\right| / 2 \sigma\right)^{a}}
$$

$a$ being characteristic of the steepness of the angular constraint from a steep edges' cone $(a \gg 2)$ to a Gaussian $(a=2)$.

Figures 3(c) and 3(d) give a polar representation of the effective distributions $p(\varphi)$ invoked in Eqs. (25) and (26), together with their reconstruction $\tilde{p}(\varphi)$ based on their second and fourth symmetry orders. These reconstructions, even though they are based on truncated circular decompositions, already reveal the essential features of the initial distributions. The behavior of the $\left(S_{2}, S_{4}\right)$ spectrum of the $p(\varphi)$ functions is shown in Figs. 3(e) and 3(f). As expected, both $S_{2}$ and $S_{4}$ values decrease when the disorder (width $\sigma$ ) increases. $S_{4}$ also is seen to be representative of high angular frequencies in the distribution function (sharp edges in a cone and distinct peaks in a bi-Gaussian). Exploiting the whole symmetry spectrum 
available from pFWM-pCARS should, therefore, provide refined information on the molecular structural behavior.

\section{EXPERIMENTAL RESULTS}

\section{A. Experiment}

This methodology is applied, as a demonstration, on MLVs and axonal myelin in mice spinal cords. MLVs were made from chain-deuterated 1,2-dipalmitoyl-sn-glycero-3phosphocholine (DPPC) lipids (DPPC-d62) [25]. Thin films, made of pure DPPC-d62 or mixed with cholesterol, were

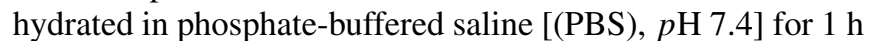
above the main phase-transition temperature $\left(45^{\circ} \mathrm{C}\right)$, leading to MLVs of 1-30- $\mu \mathrm{m}$ size in a solution enclosed between two spaced coverslips. The MLVs form almost spherical objects made of concentric multilayers of lipids. The images are performed at the equatorial plane of these objects where the distribution of the lipids is expected to be lying along the transverse sample plane. The study of the $\mathrm{CD}_{2}$ vibration is purely contextual and would lead to similar conclusions as for $\mathrm{CH}_{2}$. Myelin samples were obtained from the spinal cord of mice, were extracted, and were sectioned longitudinally into 1-mm-thick coronal acute slices bathed in PBS for imaging. In this case, the images are performed on transverse sections of myelin sheaths where the geometry is expected to be similar to MLVs with lipid distributions lying on the sample plane. At last, a system of known symmetry was used for comparison: Microcrystals of $\mathrm{NaCl}$ were deposited on a coverslip by direct spraying of a saturated salt solution.

The pFWM-pCARS microscope follows a similar scheme as described in Refs. [9,10]. Briefly, the pump and Stokes excitation beams come from two synchronized optical parametric oscillators ( $5 \mathrm{ps}, 80 \mathrm{MHz})$ with a fixed $\lambda_{p}=735-\mathrm{nm}$ pump wavelength and a Stokes wavelength set at $\lambda_{s}=884 \mathrm{~nm}$ (FWM nonresonant conditions, $2303 \mathrm{~cm}^{-1}$ ), $869 \mathrm{~nm}$ (CARS, $\mathrm{CD}_{2}$ stretching band in the MLV, $2100 \mathrm{~cm}^{-1}$ ), or $929 \mathrm{~nm}$ (CARS, $\mathrm{CH}_{2}$-stretching band in myelin, $2845 \mathrm{~cm}^{-1}$ ). The laser beams are focused in the sample at a $10-20-\mu \mathrm{m}$ depth using a [ $\times 40$ numerical aperture (NA) 1.15] objective, after reflection on a silver mirror. Images are performed by galvanometric mirrors (typically: pixel dwell time of $50 \mu \mathrm{s}, 100 \times 100$ pixels, and scan range of $30 \mu \mathrm{m}$ ) with average powers at the focal spot of $5-15 \mathrm{~mW}$. The emitted signal is collected in the forward direction by a $(\times 40$ NA 0.6$)$ objective and is directed to a photon-counting photomultiplier. The incident linear polarization angle $\alpha$ of the pump and Stokes fields is tuned from $0^{\circ}$ to $180^{\circ}$ (in $6^{\circ}$ steps) [Fig. 4(a)] by rotation of an achromatic half-wave plate mounted on a step motor at the entrance of the microscope. For each angle $\alpha$, a FWM-CARS image of the sample is recorded, leading to a stack of images [Fig. 4(b)]. The polarization distortions originating from the last mirror reflection are preliminarily characterized and are taken into account in the data analysis [with appropriate modifications of Eq. (22)].

At last, the MLVs birefringence was measured [26] through the whole MLV thickness. The birefringence phase shift is found to be of a few degrees in pure DPPC-d62 MLVs and $40^{\circ}$ on average in DPPC-d62-5\% cholesterol MLVs of 20- $\mu \mathrm{m}$ diameter.
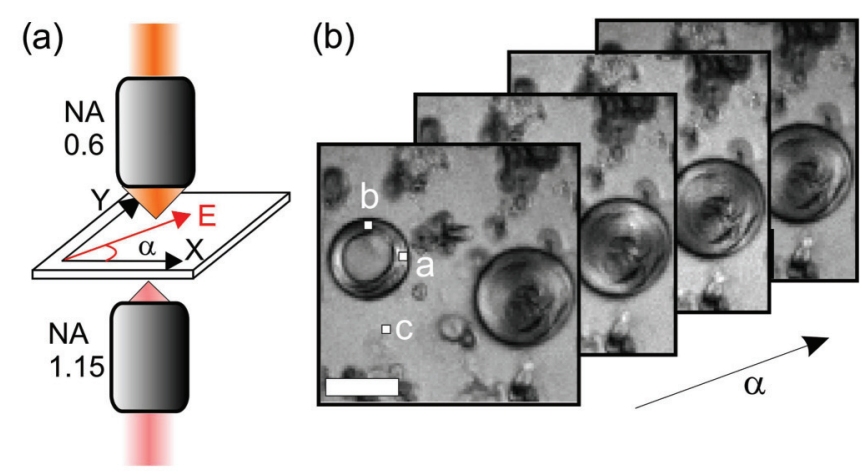

(c)

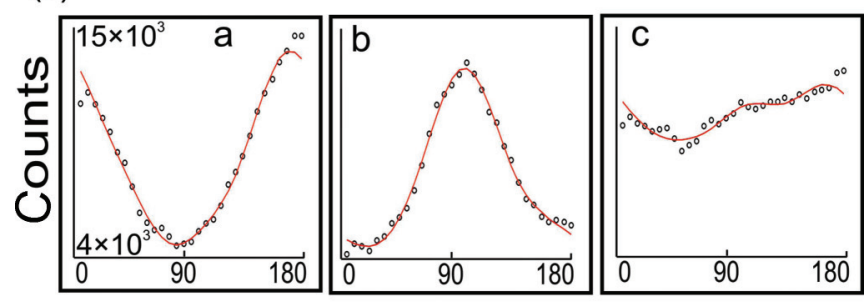

(d)
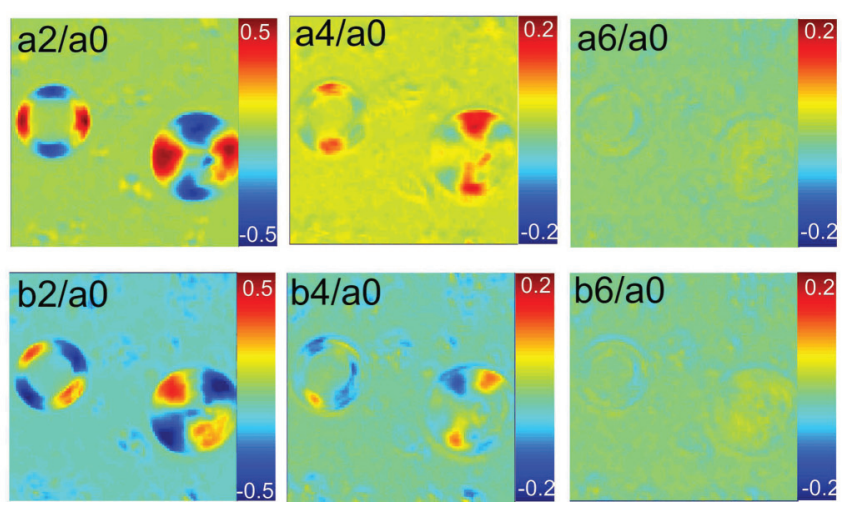

FIG. 4. (Color online) (a) Schematic of the setup geometry. (b) Stack of polarization-resolved images recorded by FWM on MLVs made of pure deuterated DPPC-d62. (c) Example of FWM polarization-resolved signals for three different pixels of the image. Left and middle: signals recorded at two different positions on the MLV, showing a significant contrast with different mean orientations. Right: signal recorded from the medium, showing the typical level of the signal from the background recorded in this experiment. (d) Images deduced from the numerical calculation of the Fourier coefficients $\left(a_{n}, b_{n}\right)$ of the polarization-resolved intensity images. Scale bar: $10 \mu \mathrm{m}$.

\section{B. Data processing}

Figure 4(c) shows the level of signal typically recorded in polarization-resolved FWM-CARS experiments on MLVs: The polarization contrast is significantly higher than that of the surrounding background. The noise level is also very low, leading to Fourier coefficients determination with estimation errors of a few percent. Resulting Fourier component $\left(a_{n}, b_{n}\right)$ images are shown in Fig. 4(d). The deduction of the $\left(p_{n}, q_{n}\right)$ order parameters from the measured $\left(a_{n}, b_{n}\right)$ coefficients relies on the nonlinear expression shown in Eq. (23). Since this nonlinear dependence makes it impossible to use an analytical 
(a)
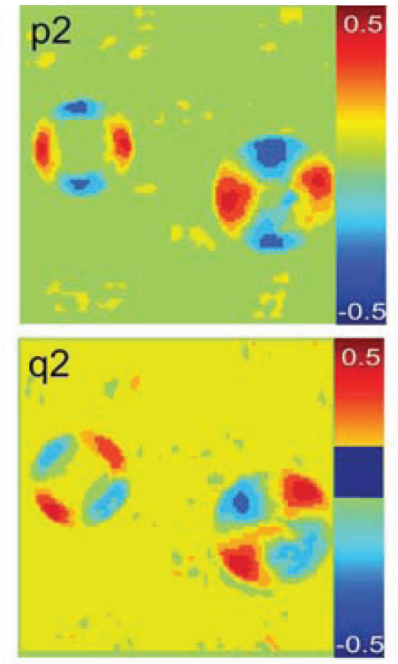

(b)

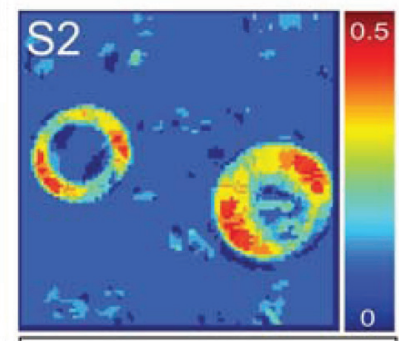

(c)

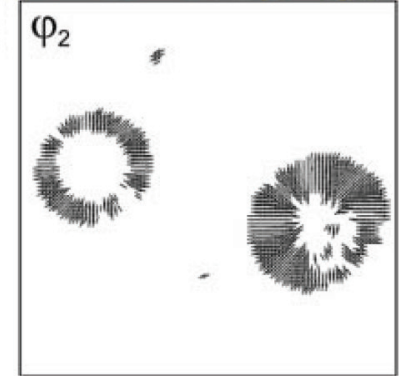

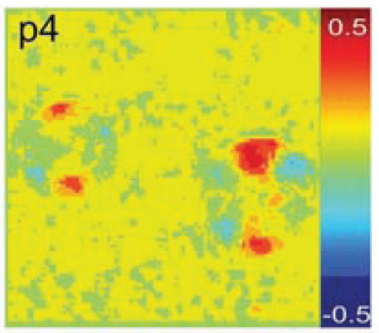
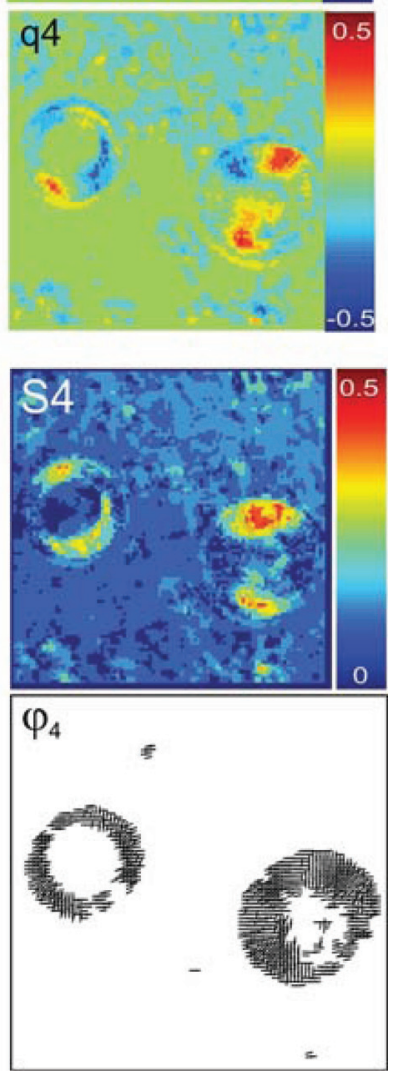

FIG. 5. (Color online) (a) Images of the molecular order parameters $\left(p_{n}, q_{n}\right)$ deduced from the sample described in Fig. 4. (b) Resulting images of global order parameters $\left(S_{2}, S_{4}\right)$. (c) Resulting images of the molecular distribution orientation angles $\left(\varphi_{2}, \varphi_{4}\right)$ materialized by the orientation of a small stick position at each pixel for which $S_{2}>0.2$.

resolution to resolve the inverse problem, we implement a procedure which consists of finding the set $\left(p_{2}, q_{2}, p_{4}, q_{4}\right)$ (with $p_{0}=1$ ) which minimizes the error function,

$$
E\left(p_{2}, q_{2}, p_{4}, q_{4}\right)=\left\|\left[w^{\exp }-w\left(p_{2}, q_{2}, p_{4}, q_{4}\right)\right]\right\|^{2},
$$

where $w^{\exp }=\left[a_{2}, a_{4}, a_{6}, b_{2}, b_{4}, b_{6}\right]$ is the set of Fourier coefficients measured and $w\left(p_{2}, q_{2}, p_{4}, q_{4}\right)$ is the expected set of Fourier components for given $\left(p_{2}, q_{2}, p_{4}, q_{4}\right)$ values. The minimization procedure allows for recovering the parameters $\left(p_{2}, q_{2}, p_{4}, q_{4}\right)$ without ambiguities on each pixel as shown in Fig. 5(a) using a sampling of $\left(p_{n}, q_{n}\right)$ within the range $[-0.5,0.5]$ with a step of 0.02 . The typical measured standard deviation is close to that expected from the noise level and reaches about 0.05 for all parameters.

From the $\left(p_{2}, q_{2}, p_{4}, q_{4}\right)$ images, the global order parameters $\left(S_{2}, S_{4}\right)$ and $\left(\varphi_{2}, \varphi_{4}\right)$ are built according to Eq. (24) [Fig. 5(b)].
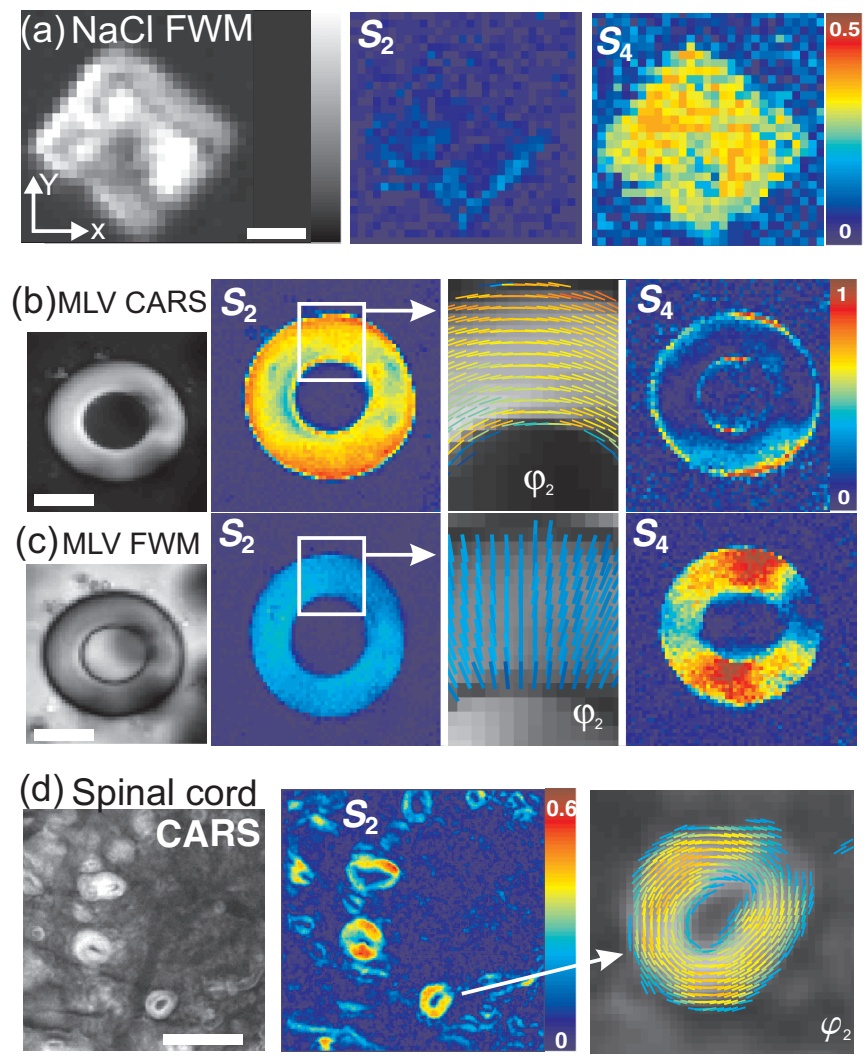

FIG. 6. (Color online) (a) Left: FWM intensity on a NaCl microcrystal. Middle and right: $S_{2}, S_{4}$ deduced images. (b) CARS image for a DPPC-d62-5\% cholesterol MLV at the $\mathrm{CD}_{2}$-stretching bond resonance. Middle and right: $S_{2}, S_{4}$ deduced images. An inset showing $\varphi_{2}$ is added, materialized by oriented sticks whose colors follow the $S_{2}$ scale. (c) FWM image of the same MLV. Middle and right: $S_{2}, S_{4}$ deduced images. (d) CARS image and $S_{2}$ image ( $S_{4}$ is omitted, giving negligible values) for myelin in a mouse spinal cord. Scale bars: $10 \mu \mathrm{m}$.

These orientation angles allow for following the average orientation of the bond dipoles' distribution in the sample plane, but also to identify a possible departure from a cylindrical symmetry. Indeed, a difference between $\varphi_{2}$ and $\varphi_{4}(\bmod [\pi / 2])$ is the signature of a distribution-shape perturbation with a loss of single-axis symmetry, breaking the symmetry of the whole distribution into a more complex one. These angles are depicted in Fig. 5(c).

\section{Results}

Typical results are shown in Fig. 6. First, the observation of the $S_{2}$ and $S_{4}$ images in the case of FWM in $\mathrm{NaCl}$ microcrystals [Fig. 6(a)] confirms the potential of this technique. Indeed, independent of the crystal orientation on the sample plane, the projection of the unit cell on this plane is found to be of pure fourfold symmetry as expected from the crystal structure. The case of MLVs made of DPPC-d62-5\% cholesterol shows remarkable features. At resonance with the $\mathrm{CD}_{2}$-stretching band [Fig. 6(b)], the $\varphi_{2}$ image shows that the nonlinear bond dipoles are found to be, on average, oriented azimuthally with respect to the MLV circular contour as expected from the lipids' geometry in a concentric arrangement. In all the 


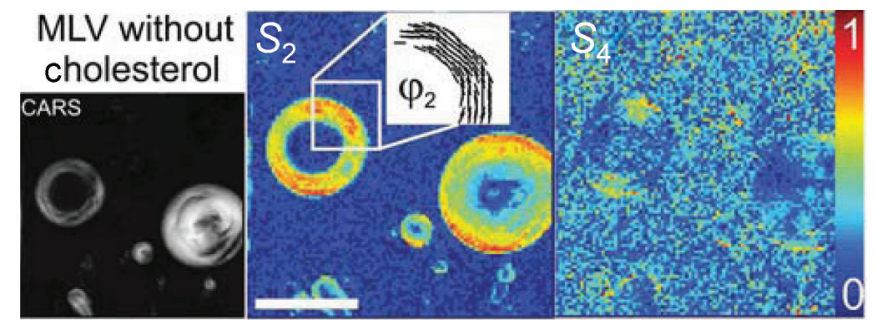

FIG. 7. (Color online) Left: CARS intensity image of MLVs made of pure deuterated DPPC-d62. The pump and Stokes wavelengths are set to address the $\mathrm{CD}_{2}$ symmetric stretching mode. Middle: corresponding $S_{2}$ image as well as a zoom on the $\varphi_{2}$ image. Right: corresponding $S_{4}$ image. Scale bar: $10 \mu \mathrm{m}$.

obtained images, $\varphi_{4}$ was found close to $\varphi_{2}$ as typical of cylindrical symmetry distributions. The $S_{2}$ and $S_{4}$ magnitudes are seen to be non-negligible with a dominant $S_{2}$ value, compatible with an angular distribution resembling a cone [Figs. 3(d) and 3(f)].

Off resonance, $\varphi_{2}$ is changed to a radial orientation [Fig. 6(c)], showing that nonlinear bond dipoles are, on average, oriented along the alkyl chain of lipids as expected from the strong nonresonant third-order susceptibility of the CC bonds. In this case, $S_{4}$ is seen to surpass $S_{2}$, emphasizing the presence of high frequencies in the angular distribution [Figs. 3(c) and 3(e)]. Note that these values, especially for $S_{4}$, are slightly underestimated due to the presence of birefringence in this sample (see above). We, nevertheless, estimated the bias on the $S_{4}$ value to be no more than 0.05 in the presence of a $20^{\circ}$ birefringence phase shift, which is the maximal value experienced here by the incident fields focused at half-height in the MLVs.

As a comparison, the analysis was performed on pure DPPC-d62 MLVs. In both FWM and CARS (Fig. 7) cases, both $S_{2}$ and $S_{4}$ values are found to be lower than in MLVs containing cholesterol, which is a signature of a global loss of order.

At last, symmetry orders of resonant lipid bonds were measured in myelin. A front view showing the concentric geometry of lipids' organization surrounding the axons [Fig. 6(d)] exhibits similar azimuthal orientation of the lipids' stretching bonds as in MLVs as expected from the tubelike packing of multiple-cell membrane layers with a visibly higher disorder shown by a smaller $S_{2}$ value and even smaller $S_{4}$ (data not shown). Note that, in all cases, the $S_{2}$ and $S_{4}$ images exhibit a high contrast with zero background (in contrast to FWM intensity images, in particular), emphasizing the capability of the technique to discriminate organized structures from their isotropic environment.

The $S_{2}$ and $S_{4}$ values measured in all samples are summarized in Fig. 8, together with expected $\left(S_{2}, S_{4}\right)$ for angular distributions of known shapes and increasing disorder $\sigma$. Obviously, none of the measured effective distributions are seen to fit with a Gaussian distribution. The pFWM data rather fit well with a bi-Gaussian made of two Gaussian functions separated by $\varepsilon=70^{\circ}$ (Fig. 3) with a visibly high disorder $\left(\sigma \approx 40^{\circ}\right)$ in pure DPPC and higher order $\left(\sigma \approx 25^{\circ}\right)$ in the presence of cholesterol. The observed symmetry spectrum of $\tilde{p}(\varphi)$ is in agreement with nonlinear bond dipoles

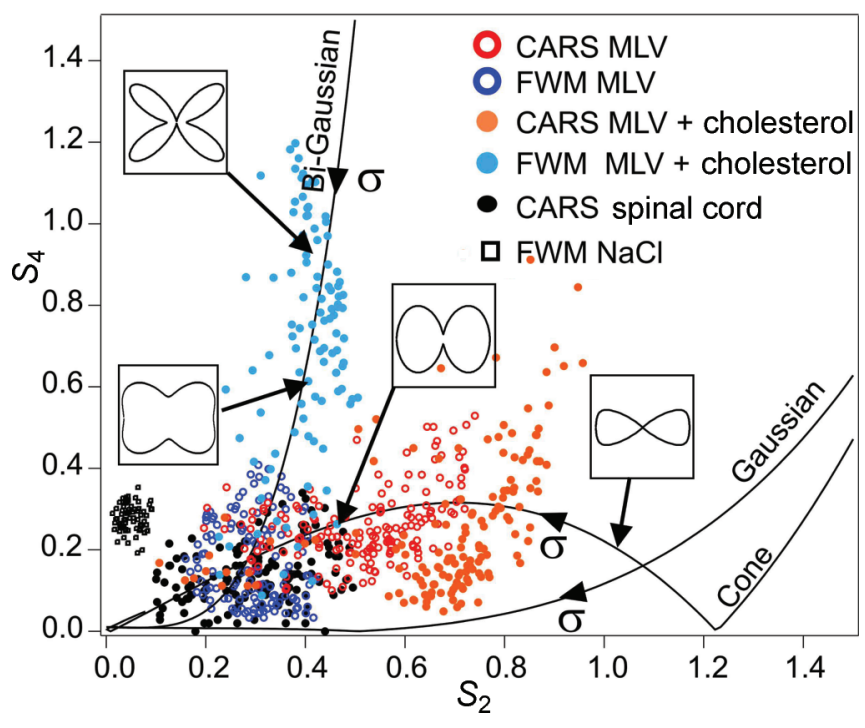

FIG. 8. (Color online) Experimental $\left(S_{2}, S_{4}\right)$ (markers are the average over ten pixels in images, such as shown in Fig. 2) for all samples described in the text. Solid lines: theoretical $\left(S_{2}, S_{4}\right)$ for a pure cubic crystal, a bi-Gaussian (distant by $\varepsilon=70^{\circ}$ ), a cone $(a=6$ ), and a Gaussian $(a=2)$ for $\sigma$ varying from $0^{\circ}$ to $180^{\circ}$. Insets: $p(\varphi)$ functions added as examples.

arranged roughly on the $\mathrm{CC}$ bonds frame, similar as in the simple theoretical model described in Fig. 2. The lower angular freedom imposed by interactions with cholesterol [17] almost reveals the crystalline structure of the individual alkyl-chain's symmetry. Note that the observed disorder $\sigma$ encompasses possible conformational changes in the lipids in the multilayers, local lipid interactions, and morphological fluctuations in the membrane at the subdiffraction scale.

The pCARS data are representative of lower $S_{4}$ values which can be interpreted by a super-Gaussian model for $p(\varphi)$, rather than by the Gaussian function usually inferred [17]. For the $\mathrm{CD}_{2}-\mathrm{CH}_{2}$-stretching bonds, the best-fitting function is a cone with a non-negligible steepness $(a \approx 6)$. The width of the observed disorder decreases in the presence of cholesterol (from $\sigma \approx 70^{\circ}$ to $\sigma \approx 45^{\circ}$ ) in agreement with previous observations [17].

At last, lipids in myelin feature the most disordered (still conelike) distribution, which can be due to a higher amount of angular freedom and morphology heterogeneities in cell lipid membranes in tissues. Note that, although some cases show quite spread $\left(S_{2}, S_{4}\right)$ values (as also reflected in Fig. 6), they still follow a single angular distribution model, which is the signature of possible sample heterogeneity in structural disorder width $\sigma$.

These results show that exploiting the $S_{4}$ parameter reveals the fine features of the lipid packing properties. Even though this information is present in all polarization-resolved thirdorder nonlinear processes, it is omitted in approaches which rely only on second-order decomposition of polarizationresolved intensities [8] or on simplified models which do not account for the full multipolar expansion of the nonlinear coupling [15-17]. The obtained effective distribution function, furthermore, permits directly picturing all nonlinear dipoles' 
contributions at the macroscopic scale without invoking $a$ priori known lipid structures and compositions. Finally, it indicates that the lipids' $\mathrm{CH}_{2}$ bonds visibly experience steep angular constraints rather than a Gaussian distribution, most probably due to space-filling conditions and a high-order interaction potential which obviously differs from a pure harmonic mean-force interaction [2].

\section{CONCLUSION}

In conclusion, we have shown that high-order symmetry information measured in polarization-resolved third-order nonlinear processes provides a refined level of analysis of the angular constraint exerted on molecular nonlinear dipoles in complex organizations, such as lipids in various membrane states. This study can be enlarged for a wide variety of molecular systems as well as other contrasts thus allowing a way for more complete structural in vivo imaging.

\section{ACKNOWLEDGMENTS}

The authors thank F. Debarbieux and G. Rougon (IBDML, France) for providing fresh spinal cord tissue to visualize the myelin sheath sample and P. Réfrégier and F. Munhoz (Institut Fresnel) for fruitful discussions. This work has been supported by Contracts No. ANR-2010-BLAN-150902 (ReceptORIENT), No. ANR-10-INBS-04-01 (France-BioImaging), and No. ANR-11-INSB-0006 (France Life Imaging) and by the Conseil Regional Provence Alpes Côte d'Azur Region. F.-Z.B. was supported by the Erasmus Mundus Doctorate Program Europhotonics (Grant No. 159224-1-2009-1-FR-ERA MUNDUS-EMJD).
[1] J. B. Klauda, N. V. Eldho, K. Gawrisch, B. R. Brooks, and R. W. Pastor, J. Phys. Chem. B 112, 5924 (2008).

[2] S. T. Nagle, Y. Liu, J. Legleiter, and J. F. Nagle, Biophys. J. 83, 3324 (2002).

[3] F. Picard, T. Buffeteau, B. Desbat, M. Auger, and M. Pezolet, Biophys. J. 76, 539 (1999).

[4] P. Stoller, B.-M. Kim, A. M. Rubenchik, K. M. Reiser, and L. B. Da Silva, J. Biomed. Opt. 7, 205 (2002).

[5] S. Psilodimitrakopoulos, S. I. C. O. Santos, I. Amat-Roldan, A. K. N. Thayil, D. Artigas, and P. Loza-Alvarez, J. Biomed. Opt. 14, 014001 (2009).

[6] J. Duboisset, D. Aït-Belkacem, M. Roche, H. Rigneault, and S. Brasselet, Phys. Rev. A 85, 043829 (2012).

[7] I. Gusachenko, Y. G. Houssen, V. Tran, J.-M. Allain, and M.-C. Schanne-Klein, Biophys. J. 102, 2220 (2012).

[8] M. Zimmerley, P. Mahou, D. Débarre, M.-C. Schanne-Klein, and E. Beaurepaire, Phys. Rev. X 3, 011002 (2013).

[9] F. Munhoz, H. Rigneault, and S. Brasselet, J. Opt. Soc. Am. B 29, 1541 (2012).

[10] F. Munhoz, H. Rigneault, and S. Brasselet, Phys Rev Lett. 105, 123903 (2010)

[11] M. Zimmerley, R. Younger, T. Valenton, D. C. Oertel, J. L. Ward, and E. O. Potma, J. Phys. Chem. B 114, 10200 (2010).

[12] S. Brasselet, Adv. Opt. Photon. 3, 205 (2011).
[13] R. P. Davis, A. J. Moad, G. S. Goeken, R. D. Wampler, and G. J. Simpson, J. Phys. Chem. B 112, 5834 (2008).

[14] Y. Fu, T. B. Huff, H.-W. Wang, J.-X. Cheng, and H. Wang, Opt. Express 16, 19396 (2008).

[15] E. Bélanger, S. Bégin, S. Laffray, Y. De Koninck, R. Vallée, and D. Côté, Opt. Express 17, 18419 (2009).

[16] G. de Vito, A. Bifone, and V. Piazza, Opt. Express 20, 29369 (2012).

[17] G. W. Wurpel, H. A. Rinia, and M. Muller, J. Microsc. 218, 37 (2005).

[18] S. Brasselet and J. Zyss, J. Opt. Soc. Am. B 15, 257 (1998).

[19] J. H. Christie and D. J. Lockwood, J. Chem. Phys. 54, 1141 (1971).

[20] B. Hudson, J. Chem. Phys. 61, 5461 (1974).

[21] J. X. Cheng, S. Pautot, D. A. Weitz, and X. S. Xie, Proc. Natl. Acad. Sci. USA 100, 9826 (2003).

[22] B. Richards and E. Wolf, Proc. R. Soc. London, Ser. A. 253, 358 (1959).

[23] E. Y. S. Yew and C. R. J. Sheppard, Opt. Express 14, 1167 (2006).

[24] P. Schön, M. Behrndt, D. Aït-Belkacem, H. Rigneault, and S. Brasselet, Phys. Rev. A 81, 013809 (2010).

[25] A. D. Bangham, M. W. Hill, and N. G. A. Miller, in Methods in Membrane Biology, Vol. 1 (Springer, US, 1974), p. 1.

[26] D. Aït-Belkacem, A. Gasecka, F. Munhoz, S. Brustlein, and S. Brasselet, Opt. Express 18, 14859 (2010). 